


\title{
¿Vivimos en un lugar seguro? Percepción de criminalidad en la Zona de Occidente ${ }^{1}$
}

Do we live in a safe place? perception of criminality in the western area

\author{
Juan Diego Carcía-Castro² Marianela Villalobos Ulate ${ }^{3}$ \\ Recibido: 16/10/2015 / Aprobado: 1/11/2016
}

Resumen

\begin{abstract}
Se presentan los resultados de una investigación que busca contribuir en el estudio de la percepción de la criminalidad en la zona de Occidente de Costa Rica, se exploran las opiniones de vecinos que han sido víctimas de actos criminales contra la propiedad, con la idea de conocer la percepción sobre el lugar de residencia, los principales problemas de la zona de Occidente, la seguridad pública? y los lugares u ocasiones considerados como inseguros. Para ello, se realizó una investigación descriptiva y cualitativa con seis personas. Dentro de los resultados destacan que en general se percibe una gran desconfianza en la seguridad pública, Los principales problemas percibidos son: la delincuencia, la drogadicción y los barrios marginales. Se discuten las implicaciones de los resultados para el estudio de la percepción de la criminalidad y para la construcción de las subjetividades en la zona de Occidente.
\end{abstract}

Palabras Claves: criminalidad, lugar de residencia, ocasiones inseguras, seguridad pública y Zona de Occidente.

\section{Abstract}

The article presents the results of a study that pretends to perceive criminality in the west area of Costa Rica, based on the residents' opinions of a region where some people have been offended in criminal acts against property. The question addressed to participants was: what is the perception about the neighborhood? Which are the main problems? Which places and situations are perceived as unsafe? A descriptive and qualitative research was developed with six people. Results show that there is a general perception of distrust in public security, as one of the major problems. It is suggested that changes in its mechanisms should be done. However, it is perceived a sense of security regarding this locality early, but unsafe in dark and solitary places. Delinquency, drug addiction and marginal areas are some of the main problems perceived. The outcomes show a perception of criminality and a construction of subjectivities in this area that may have implications in the future.

Key words: criminality, place of residence, insecure occasions, public security and west area.

\footnotetext{
${ }^{1}$ El artículo es producto de la investigación 540-B3-320 “Criminalidad en Costa Rica: clases sociales, ideología y victimización. Un análisis psicosocial”, financiada por la Sede de Occidente y la Vicerrectoría de Investigación de la Universidad de Costa Rica.

${ }^{2}$ Magister en Psicología de la intervención social y estudiante del doctorado en Psicología de la Universidad de Granada, España. Profesor e investigador de la Universidad de Costa Rica, Sede de Occidente. Contacto: juandiego.garcia@ucr.ac.cr

${ }^{3}$ Bachiller en Psicología. Candidata a la licenciatura en Psicología por la Universidad de Costa Rica, Sede de Occidente. Contacto: marianela.villalobos_u@ucr.ac.cr
} 


\section{Introducción}

En San Ramón hubo 222 robos a viviendas durante el 2015, con lo cual se ubicó como el octavo cantón con mayor tasa de este tipo de crimen en el país (OIJ, 2015). Sin embargo, sus habitantes consideran el cantón como seguro. Datos como estos, evidencian que la Zona de Occidente resulta de especial interés científico para conocer cómo se experimenta la victimización criminal en personas que la han vivido en su propia comunidad.

El panorama latinoamericano se encuentra permeado por el aumento de la violencia, la criminalidad, la sensación de inseguridad ciudadana, así como una inadecua $\neg$ da respuesta real o percibida por parte de los organismos competentes en la materia y de la sociedad civil. Todas estas problemáticas repercuten en factores tanto económicos, políticos, sociales y psicológicos de las personas (Kliksberg, 2008).

La inseguridad y en especial, la percepción de inseguridad por parte de los ciudadanos, es uno de los grandes problemas sociales. Durante las últimas décadas, el aumento de la delincuencia que enfrentan diversas ciudades del mundo y en especial en América Latina ha sido tema central de debate tanto a nivel de opinión pública como de investigaciones realizadas por diferentes disciplinas de las ciencias sociales (Morquecho y Vizcarra, 2008).

La percepción de criminalidad se entiende como el nivel de desconfianza hacia supuestos actos delictivos. Surge a partir de experiencias personales, historias de personas conocidas o desconocidas y de los medios de comunicación (Cisneros y Zubillaga, 1997).

A pesar de ello, el discurso de la criminalidad no corresponde necesariamente con la experiencia real. Es parte de un discurso ideológico que justifica en favor de la clase dominante, mayor inversión en políticas de control social como inversión económica en la policía, patrullaje internacional en los mares, mayor presencia de la fuerza pública en la calle y en los lugares que habitan los (as) pobres (Huhn, 2008).
Este artículo presenta los resultados de una investigación que busca contribuir en el estudio de la percepción de la criminalidad en la zona, mediante las opiniones de quienes habitan en la zona. Se planteó conocer: ¿Cuál es la percepción del lugar de residencia?, ¿Cuáles son los principales problemas de la zona de Occidente?, ¿Cuál es la percepción de la seguridad pública?, y ¿Cuáles lugares u ocasiones son percibidos inseguros? Para ello, se realizó una investigación descriptiva y cualitativa con seis personas víctimas de crimines contra la propiedad.

\section{Percepción del lugar en el que vivimos}

La percepción del lugar en el que se vive es un tema de larga data en la Psicología social. Se conoce que el percibir vivir en un lugar agradable y seguro brinda bienestar psicológico, por el contrario, la percepción de vivir en un lugar desagradable, sucio y feo genera malestar (Acuña-Rivera, Browm y Uzzell, 2014).

Dentro de las variables que inciden en la percepción del lugar en el que se vive destacan la clase social de las personas que conforman la comunidad, la etnia y el haber sufrido anteriormente una victimización criminal en lugar. A esto se le conoce como la teoría de la "ventana rota", la cual postula que la percepción del lugar de residencia puede aumentar o disminuir la incidencia de actos criminales (Franzini, O’Brien, Murray y O'Campo, 2008).

Las señales físicas del entorno inciden en la percepción sobre la realidad. Por ejemplo, vivir en lugares con basura en la calle, grafitis, con falta de iluminación o casas en mal estado inciden en el miedo con el que cuentan las personas. Así como los comportamientos no normativos como la prostitución, tomar licor en espacios públicos o la indigencia (Pérez, 2012).

\section{Percepción de problemáticas sociales}

En América latina, con lo que respecta al tema de la percepción acerca de los principales problemas que puede tener un país, se destaca una encuesta realizada en el 2007 en distintos países, en donde se preguntó cuál es el problema más importante del lugar en el que la persona vive. Se encontró que 
la inseguridad, la pobreza, la falta de acceso a la salud y la educación, la corrupción y el desempleo, son las grandes preocupaciones de la población. En el caso de Costa Rica destacó como el principal problema la delincuencia (Kliksberg, 2008).

También mediante una investigación realizada por Huhn en el 2008, en Costa Rica, Nicaragua y El Salvador, acerca de la percepción de la inseguridad, se les solicitó a estudiantes de una significativa cantidad de colegios tanto públicos como privados, que escribieran acerca de las principales problemáticas sociales percibidas; sin haberles preguntado por ello directamente y con la posibilidad de elegir cualquier otro tema, el 68 por ciento de los alumnos en Costa Rica nombraron la violencia y la criminalidad como el problema social más grave.

La "Política integral y sostenible de seguridad ciudadana y promoción de paz social" que corresponde al plan de seguridad ciudadana del gobierno 2010-2014 en Costa Rica, asegura que en los últimos estudios de opinión pública se destaca que los costarricenses manifiestan gran preocupación por el tema de la inseguridad ciudadana, considerándolo el principal problema que tiene el país, incluso mayoritariamente que los económicos (Programa de las Naciones Unidas para el Desarrollo, PNUD, 2009-2010).

\section{Percepción de seguridad pública}

Otro factor relacionado con la percepción de la inseguridad es la desconfianza a la seguridad pública. Por ejemplo, Vilalta (2010) encontró que las personas presentan mayor miedo al crimen cuando no hay confianza en la policía y, contrario a esto, cuando el nivel de confianza en el sistema policial es mayor, las personas se sienten más seguras y su percepción de inseguridad y el miedo al crimen disminuye.

En otra investigación en la que se comparaban las percepciones ciudadanas sobre la policía en Buenos Aires, Argentina y el Distrito Federal, en México se mostró que en ambas ciudades se posee una percepción devaluada de la policía. Quienes consideran un trabajo eficiente, se muestran con mayor disposición en confiar en ella; sin embargo, este representa un grupo minoritario, a diferencia de los que creían que la policía era deficiente en sus tareas. De igual forma concluyeron que hay algunas variables que afectan la confianza en la policía como el estrato social, la edad, el género y la victimización previa sobre las cuales la policía no puede "operar" en demasía (Bergman y Flom, 2012).

En el Latibarómetro realizado en el 2007 se muestran altos niveles de desconfianza a las instituciones públicas, se indica que solo un 23 por ciento de la población destaca sentirse protegida ante el crimen. El 31 por ciento no le tiene ninguna confianza a la policía, el 29,8 por ciento le tiene poco, el 24,9 por ciento un poco y solo el 12 por ciento le tiene confianza (Salame, 2008).

Otra investigación en la cual se recogieron datos acerca del tema de la criminalidad de igual forma en diferentes países de Latinoamérica, analizando encuestas realizadas desde 1996 hasta el 2010, muestran que la confianza en los policías osciló entre el 29 por ciento y el 39 por ciento, con un promedio anual del 34 por ciento. Se trata de niveles de confianza muy bajos, pues alrededor de las dos terceras partes de la población latinoamericana entre el 61 por ciento y el 71 por ciento expresaron de manera sostenida poca o ninguna confianza (Costa, 2012).

Por otro lado, Medina (2003) indagó acerca de la inseguridad ciudadana y el miedo al delito en España, mediante encuestas, con el fin de arrojar datos sobre los factores ecológicos e individuales que inciden en la problemática. Sobre la presencia policial en la calle y su relación con el miedo al delito, el estudio demostró que este no tuvo un efecto significativo en la reducción del miedo al delito, pero si un ligero efecto en una mejor imagen ciudadana de la policía.

En otro estudio realizado en Argentina por Dammert y Malone (2002), buscaban analizar sobre los factores que causan la percepción inseguridad pública en la población, estimando mediante los resultados que la confianza en la policía, corrupción, y la presencia del crimen violento son una primera explicación a los niveles de inseguridad ciudadana. 


\section{Percepción de ocasiones inseguras}

Diversos estudios han indagado acerca de cuáles son las principales percepciones de lugares u ocasiones inseguras. Pérez (2012) menciona que los lugares que son considerados peligrosos influyen en la percepción de inseguridad, ya que estos espacios se caracterizan por una falta de iluminación, la existencia de sombras y elementos ambientales o arquitectónicos que disminuyen el dominio visual del espacio. Además de estos factores ambientales, la percepción de la criminalidad también va a estar incidida por diversas variables personales como lo son las experiencias previas, percepción de vulnerabilidad y las psicosociales (satisfacción residencial, identidad urbana).

En un estudio en España se planteó conocer los lugares temidos, el significado para el individuo, así como sus respuestas comportamentales y afectivas, mediante la realización de entrevistas. Los resultados apuntan que la mayoría de sujetos señalan como indicadores del miedo al delito, aspectos relacionados con la apariencia física del lugar, como lo es el deterioro, la baja iluminación y dificultades visuales. De igual forma se menciona como relevante la presencia de individuos ya sea posibles ofensores o algún tipo de apoyo (Fernández y Corraliza, 1997).

Por su parte, en una encuesta de percepción y victimización realizada en Bogotá, Colombia en el 2009, se pudo hallar que el 39 por ciento de los encuestados percibe las calles como los lugares más inseguros de la ciudad. El 60 por ciento de los ciudadanos encuestados señala que tal sensación está determinada por la presencia de grupos (delincuentes y pandillas juveniles). El restante 40 por ciento la relaciona con factores ambientales (soledad y oscuridad) (Cámara de Comercio de Bogotá, 2009).

En síntesis, los estudios realizados acerca de los principales problemas percibidos en Costa Rica, apuntan hacia el tema de la violencia y la criminalidad mayoritariamente (Hunh, 2008). Sin embargo, no se ha indagado en profundidad la percepción de la población más allá del Valle Central. También a través del análisis de la bibliografía se revela que la desconfianza a la policía influye para la generación del miedo al delito. Además de presentarse porcentajes de desconfianza altos a nivel latinoamericano (Costa, 2012). En cuanto a los estudios relacionados con la percepción de ocasiones y lugares inseguros, se remite mayoritariamente a aspectos físicos y ambientales, caracterizados primordialmente por un tema de dominio visual (Pérez, 2012).

Esta investigación partió de la hipótesis que sostiene que las principales problemáticas percibidas serían la criminalidad, con un mayor énfasis en el tema de la delincuencia, teniendo influencia la experiencia criminal vivida anteriormente por los participantes. Asimismo, se presentaría desconfianza hacia la policía. Además de percibir los lugares oscuros y solitarios como inseguros.

\section{Metodología}

El presente trabajo utilizó un diseño descriptivo y cualitativo de entrevistas a profundidad con mujeres y hombres de la zona de Occidente que fueron víctimas durante el último año de crimines contra la propiedad. Este tipo de metodología ha sido utilizada en otras investigaciones, siendo el discurso obtenido mediante la entrevista, la principal herramienta de análisis para la obtención de los resultados (Cisneros y Zubillaga, 1997; Huhn, 2008).

\section{Participantes}

Se llevaron a cabo 6 entrevistas, 3 hombres y 3 mujeres, con edades productivas entre los 22 y 55 años. Provenientes y habitantes de la zona de Occidente, específicamente de San Ramón y Grecia quienes se dedican a estudiar, trabajar para empresas privadas o son comerciantes. Todos los participantes fueron víctimas durante el último año de crímenes contra la propiedad, asaltos a mano armada en la calle o robo a su casa de habitación sin estar presentes.

Los participantes fueron contactados a través de referencias de personas conocidas quiénes respondieron a una convocatoria realizada en la Universidad de Costa Rica, Sede de Occidente (San Ramón) para remitir información de personas víctimas de actos criminales. Se contactaron a través de llamada telefónica o de correo electrónico $\mathrm{y}$ accedieron a participar de forma voluntaria. 
Se utilizó un consentimiento informado, que fue explicado de manera previa a la realización de la entrevista, se dejó claro que la participación era voluntaria, anónima y sin adquisición de ganancia económica o de algún tipo y que en caso de sentirse incomodos podían desistir de la entrevista. Nadie desestimo su participación.

\section{Procedimiento}

En primera instancia se realizó una convocatoria para remitir información de personas que hayan sido víctimas de un acto criminal. Con los datos obtenidos se contactó a las personas para conocer su voluntad de participación y concertar una cita para realizar la entrevista. Las entrevistas fueron transcriptas en su totalidad y se procedió a realizar el análisis.

\section{Entrevistas}

Las entrevistas se ejecutaron dentro de las instalaciones de la Universidad de Costa Rica, Sede de Occidente, en la casa de las personas o en lugares acordados como bibliotecas públicas. Fueron llevadas a cabo por uno o dos de los investigadores principales. En promedio tuvieron una duración de 45 minutos. Algunas de las preguntas que se realizaron fueron: cuéntenos de la experiencia sufrida, ¿En qué lugares se siente seguro e inseguro? y ¿En general, cómo se siente en el lugar en el que vive?

\section{Análisis}

El análisis se realizó a través del método de comparación constante. En primera instancia se transcribieron completamente las entrevistas. Luego, se revisaron y con las ideas principales que se repetían se construyeron categorías. Una vez que la información se había codificado, se describieron los ejemplos prototípicos de cada una. Finalmente, las categorías se contrastaron para clasificar de forma precisa toda la información (Ralph, Birks y Chapman, 2015).

Se buscaron cuatro expertos en el tema para verificar si las construcciones de las categorías y su interpretación les parecían correctas buscando mantener la validez de la información. En su totalidad validaron y confirmaron los resultados.

\section{Resultados}

Se presentan a continuación los resultados más importantes en relación con diversas temáticas abordadas en las entrevistas. A saber, percepción sobre el lugar de residencia, los principales problemas de la zona de Occidente, la percepción de los participantes acerca de lugares y ocasiones inseguras y de último la percepción sobre la seguridad pública.

\section{Percepción del lugar de residencia}

En este aspecto se presentan algunas respuestas relacionadas con un sentimiento de seguridad en la zona de Occidente. La mayoría de entrevistados mencionó que donde viven es tranquilo, por ejemplo, una de las participantes respondió "este (...) no yo me siento bien es tranquilo" (Anónimo, comunicación personal, 23 de julio, 2014). Sin embargo, adicionalmente explican que con el paso de los años han surgido cambios en la zona que han propiciado, primero, un sentimiento de inseguridad y segundo, que se tomen medidas de precaución; "yo siento que de un tiempo para acá las cosas han cambiado en un porcentaje muy alto, porque se ve mucho más, este, diay la delincuencia" (Anónimo, comunicación personal, 23 de julio, 2014).

Asimismo, uno de los participantes explicó que a través de los distintos mecanismos de seguridad privada se logra una sensación de seguridad, aunque no es total; "con ciertos obstáculos que, que ponemos para, para salvarnos como las verjas y, algo así parecido pues, este, nos sentimos en un buen porcentaje de seguridad (...) no en un 100 por ciento" (Anónimo, comunicación personal, 6 de agosto, 2014).

\section{Principales problemas de la zona de Occidente}

En cuanto a la percepción de los principales problemas de la zona de Occidente, tres de los participantes respondieron que poca seguridad, asociándolo en su mayoría con asaltos, por ejemplo una de las personas menciona: "yo siento que hasta cierto punto lo que es el hurto, en cuanto a que, o sea es un punto en que las personas viajan a San José o vienen de Puntarenas o buscan, no sé, viajar hacia San Carlos o algo así, entonces muchas personas 
se descuidan muchísimo, entonces he escuchado cualquier cantidad de historias de personas en los buses que les quitan lo que traen y ni siquiera se dan cuenta" (Anónimo, comunicación personal, 22 de Abril, 2014); "di asaltos por cualquier lado, hasta en la puerta de su casa” (Anónimo, comunicación personal, 6 de Agosto, 2014).

De igual forma, tres de los entrevistados, comentan que otra de las problemáticas presentadas es el consumo de drogas; "el aumento del consumo de drogas, eso definitivamente, ha empeorado la situación y nosotros, hasta cierto punto lo hemos comentado que, que esto, eh va en aumento y entonces aumenta también la, la vagabundería la manera de que ellos de alguna forma tienen que conseguir la droga" (Anónimo, comunicación personal, 24 de julio, 2014), "yo sé que hay mucha droga por ejemplo en San Ramón (..)" (Anónimo, comunicación personal, 25 de julio, 2014).

Por otra parte, dos de las personas entrevistadas mencionan que los barrios marginales son otro problema presente en la zona; “(..) hay algunos barrios así como muy marginados” (Anónimo, comunicación personal,24 de julio, 2014). Se expresa incluso que dicho tipo de barrios pueden generar una sensación de temor importante, por lo que se tiende a evitar el transitar por lugares de este tipo. Por ejemplo, una de las entrevistadas se refirió a un barrio considerado peligroso diciendo: "toda la gente, ahí son delincuentes el papá, la mamá, los hijos, la suegra, el suegro y todo el mundo, entonces la inseguridad es, muchísima" (Anónimo, comunicación personal, 24 de julio, 2014).

\section{Percepción de la seguridad pública}

En relación con las opiniones de los participantes con respecto a la policía pública costarricense, la mayoría se inclinaron por un definitivo "no" cuando se les preguntó si confían o no ella. Además, se encontraron comentarios respecto a la fuerza pública como los siguientes: "Sinceramente no (...) los lineamientos que tienen son buenos (...) las condiciones en las que están no son las mejores (...); sin embargo, yo siento que hay una falta de, de disposición del cuerpo policial en realidad al estar al servicio de la comunidad" (Anónimo, comunicación personal, 22 de abril, 2014). Por otra parte, se mencionó: "realmente, no podemos confiar (...) ni en un 60 por ciento, ¿por qué? porque hemos visto, a través de las noticias (...) que dentro de la policía también hay delincuentes" (Anónimo, comunicación personal, 6 de agosto, 2014).

Sin embargo, hubo dos opiniones en donde no se plasmó una rotunda desconfianza hacia la policía, sino más bien consideraron que deben llevarse a cabo cambios en esta. Por ejemplo; "se debería de mejorar un poco en la disciplina que tienen (...) hay muchos que sí hacen su trabajo, pero otros (...) no lo hacen ¿por qué?, por vagos, o porque ya confían como ya tengo el puesto, me van a seguir pagando" (Anónimo, comunicación personal, 23 de julio, 2014).

\section{Percepción de ocasiones inseguras}

Con respecto a los lugares inseguros, se destacó que los que son solitarios y oscuros provocan una mayor sensación de inseguridad. Asimismo, los participantes expresaron que especialmente durante la noche, "uno se siente más indefenso en las noches", sin importar el lugar, existe un temor mayor a ser víctimas de un acto criminal. Sin embargo, también se presentaron respuestas en las que se destaca que -en la actualidad- no existen lugares plenamente seguros y que la amenaza siempre es latente. Por ejemplo: "ahora asaltan a cualquier hora y cualquier lugar, incluso en el hogar" (Anónimo, comunicación personal, 24 de julio, 2014), "no hay lugar seguro" (Anónimo, comunicación personal, 24 de julio, 2014), "entonces en la calle, aunque ande con personas siempre ando un poco ahí desconfiada" (Anónimo, comunicación personal, 6 de agosto, 2014). De igual forma se presenta mayor confianza en lugares "conocidos" o con persona que representen confiabilidad para las participantes, a diferencia de lugares donde no se posee conocimiento de su dinámica, por ejemplo: “yo me siento segura si yo ando con personas (...) con más gente que sean de mi confianza verdad, amigos de mi confianza y así" (Anónimo, comunicación personal, 6 de agosto, 2014), "uno se siente muy seguro (...) y en barrios que uno conoce (...) inseguro 
también en barrios donde uno no conoce porque (...) diay no sabe que (...) qué esperar de ahí (...) ya cuando uno lo va conociendo se va sintiendo más seguro (Anónimo, comunicación personal,6 de agosto, 2014)". Además, se encuentra cierta en inseguridad en los llamados "barrios marginales"; por ejemplo: "también en los barrios marginados (...) así porque ahí suele darse mucha delincuencia entre los mismos barrios y todo entonces entrar a esos barrios es un poco de inseguridad para uno porque (...) uno está acostumbrado a estar seguro y todo y saber que ahí es donde se origina el problema tal vez a uno le da inseguridad o tal vez miedo entrar ahí a que le pase algo" (Anónimo, comunicación personal, 23 de julio, 2014).

\section{Discusión}

Dentro de los resultados encontrados se puede mencionar que en general se percibe la criminalidad como uno de los principales problemas en la zona y una inseguridad generalizada. Un nivel alto de desconfianza en la seguridad pública, planteado a su vez como un problema de relevancia dentro del área. Se presenta desconfianza en lugares oscuros y solitarios y una contradicción entre la percepción segura del lugar de residencia y la inseguridad latente.

Los principales problemas que se perciben en la comunidad son la criminalidad, asociado principalmente a los asaltos, la drogadicción es el segundo problema planteado y luego los barrios marginales. Es decir, la criminalidad representa una problemática alarmante para dicha población, estando además permeada dicha percepción por la experiencia previa del acto criminal (Pérez, 2012).

De igual forma se confirman los datos hallados en las encuestas latinoamericanas que presentan el crimen como uno de las principales preocupaciones de nuestros habitantes. Según los estudios realizados por Hunh (2008) y Klisberg (2008) la criminalidad y la violencia son consideradas las problemáticas más preocupantes de América Latina. Así como los indicadores en el 2012 y 2013 que catalogan al cantón de San Ramón en una posición alta en cuanto al hurto y robo de vivienda (OIJ, 2013).

Las entrevistas, evidencian que la confianza hacia la seguridad pública es baja y ésta es planteada como una de las problemáticas mayores en la cual se debe buscar mejorías. Es decir, los resultados apuntan a que la desconfianza en la policía se convierte en una posible explicación para el tema de la inseguridad ciudadana, ya que, a pesar de sentirse seguros en el lugar de residencia, prevalece el sentimiento de inseguridad, percibiendo desprotección en las instituciones encargadas de controlar el crimen. Estos datos coinciden con los hallazgos en las investigaciones de Dammert y Malone (2002) y Vilalta (2006).

Además, se presenta una asociación directa con su propia experiencia criminal y la percepción de deficiencia en la labor policial. Es decir, estos resultados conllevan a creer que una mayor confianza en la seguridad pública, va a ayudar a disminuir el miedo y la percepción de inseguridad que las personas manifiestan; en tales datos se evidencia que esta es un área de estudio competente para la disminución del miedo en la población.

Se percibe un mayor miedo en lugares solos y oscuros, relacionados mayoritariamente con la noche, a diferencia de los lugares donde típicamente suceden los actos criminales. Sin embargo, el sentimiento de inseguridad se percibe de forma constante; se presenta -por ende- una representación compartida en cuanto a los lugares u ocasiones percibidas como inseguras -según predomina en la percepción- ocasiones o lugares donde no se presenta un total dominio visual del espacio y se percibe "deshabitado", como bien es mencionado en el trabajo de Fernández y Corraliza (1997) y Pérez (2012), a diferencia de los datos encontrados por Hunter (1978) citado por Pérez (2012), donde señales como grafitis o daño inmobiliario eran indicadores de percepción de criminalidad.

Adicionalmente, en lo que concierne al lugar de residencia, las respuestas de los entrevistados se inclinan hacia un sentimiento de seguridad, rescatando; sin embargo, que durante los últimos años se han presentado algunos cambios como lo es el aumento de la criminalidad. 
Los participantes manifiestan que su comunidad es segura a pesar de haber sido victimizados en ella. La ideología y la percepción de su espacio superan la experiencia real. Se plantea que esta contradicción resguarda la subjetividad de la amenaza constante de inseguridad y se manifiesta como un mecanismo de autoprotección, ante la angustia y el malestar de considerar la posibilidad constante de ser víctima de un acto criminal. Se percibe de esta manera el lugar de residencia, como un lugar agradable, con lo cual se genera mayor bienestar psicológico; estos resultados se apoyan también en investigaciones anteriores (AcuñaRivera, Browm y Uzzell, 2014).

Por otro lado, se evidencia que un mayor conocimiento del lugar implica una percepción de mayor control de su ambiente, como son los "barrios" conocidos o sus propios "barrios". Lo cual genera sentimientos de seguridad, al igual que con personas que figuran confianza como los "amigos" o espacios físicos como la casa.

\section{Conclusiones}

Los resultados apuntan a que en general se presenta una inseguridad latente en los participantes, donde la criminalidad es percibida como una de las problemáticas más graves actualmente y considerada alarmante en la sociedad. Además de niveles altos de desconfianza en la seguridad pública al mostrarse como indicador de la inseguridad percibida. Así como la percepción de lugares inseguros como lugares oscuros y solitarios, en concordancia con la teoría analizada. El lugar de residencia es percibido como seguro, en contradicción con la inseguridad latente, de ahí que se plantea como un mecanismo de autoprotección para el bienestar psicológico. Se concluye -por ende- que la inseguridad generalizada que presentan los participantes, se encuentra asociada a la experiencia criminal vivida, la desconfianza policial y la percepción de la criminalidad como uno de los problemas más alarmantes.

Tales hallazgos son de suma relevancia para la Zona de Occidente, ya que indican parámetros para futuras intervenciones en la problemática, fortalecimiento de algunas áreas relacionadas con el tema de la seguridad pública y la búsqueda del bienestar psicológico y social de la población; de ahí que se considera un desafío importante seguir estudiando estas problemáticas en contextos latinoamericanos y profundizar investigaciones en nuestro país, creando conciencia de la importancia del impacto que el miedo al crimen puede tener en las sociedades.

Se conocen hasta el momento las consecuencias psicológicas y sociales de la criminalidad y el miedo a la criminalidad, así como su instrumentalización de parte de los sectores dominantes; sin embargo, la investigación en América Latina todavía es escasa en el tema de reducción del miedo en la población, la cual podría contribuir de manera importante en la búsqueda del bienestar psicológico. Esta es la principal deuda pendiente y uno de los principales caminos hacia donde se debe dirigir su estudio.

Una de las limitaciones presentes en la investigación, es la especificad del contexto donde se realiza el trabajo por lo que presenta mayor dificultad para trasladar los resultados a otras realidades. Además, es relevante recalcar que el estudio es propiamente de tipo descriptivo y con personas que fueron víctimas directas de la criminalidad.

Es importante destacar que este tipo de investigaciones son de gran necesidad, ya que más allá de los datos estadísticos que se indagan sobre el tema de la criminalidad, es necesario contextualizar el problema y asumir con responsabilidad el miedo y la percepción de la inseguridad que la sociedad está manifestando.

Finalmente, cabe mencionar que la información hallada a través de la investigación presenta indicadores claves para el accionar en la zona de Occidente. La percepción de la criminalidad como un problema latente, la desconfianza hacia la policía y los sentimientos de inseguridad generalizada, son desafíos para la búsqueda de disminución del miedo y el bienestar psicológico de la población. 


\section{Referencias}

Acuña-Rivera, M., Brown, J., y Uzzell, D. (2014). Risk perception as mediator in perceptions of neighbourhood disorder and safety about victimisation. Journal of Environmental Psychology, 40, 64-75. doi: 10.1016/j. jenvp.2014.05.002

Bergman, M., y Flom,H. (2012). Determinantes de la confianza en la policía: una comparación entre Argentina y México. Perfiles Latinoamericanos, 40, 97-122. http://www.redalyc.org/articulo. oa?id=11523037004

Cámara de Comercio de Bogotá. (2009). Encuesta de Percepción y Victimización: Bogotá y Localidades. http://bibliotecadigital.ccb.org. co/handle/11520/3035

Cisneros, Á., y Zubillaga, V. (1997). La violencia desde la perspectiva de la víctima: la construcción social del miedo. Espacio abierto, 6(1), 7197. http://www.produccioncientificaluz.org/ index.php/espacio/article/view/2384

Costa, G. (2012). La Situación de la Seguridad Ciudadana en América Latina. Revista electrónica de derechos humanos, 31,2-14.http:// www.google.com/url? sa $=t \& r c t=j \& q=\&$ esrc $=$ s\&source $=$ web\&cd $=2 \& v e d=0 \mathrm{CCcQFjAB} \& u$ $\mathrm{rl}=\mathrm{http} \% 3 \mathrm{~A} \% 2 \mathrm{~F} \% 2 \mathrm{Fwww}$. latinobarometro. org\%2Fdocumentos\%2FLATBD_La_ seguridad_ciudadana.pdf\&ei=QS5xVeeTJIi dgwTl24HoBw\&usg=AFQjCNFw1D8i4m8 Fvh-eJf9M7BWQgCPhEA\&sig2=hvgP83p5 1HvNu1z8vxagSA\&bvm=bv.95039771,d.eXY

Dammert, L., y Malone,M. (2002). Inseguridad y Temor en la Argentina: el impacto de la confianza a la policía y la corrupción sobre la percepción ciudadana del crimen. Desarrollo Económico, 42 (1), 285-301. http://www. academia.edu/2401034/Inseguridad_y_ temor_en_Argentina
Fernández, B., y Corraliza, J, A. (1997). Hacia una tipología de lugares peligrosos en relación con el miedo al delito. Psychosocial Intervention, 6 (2), 237-248. http://dialnet.unirioja.es/servlet/ articulo? codigo $=2012648$

Franzini, L., O’Brien, M., Murray, S., y O’Campo, P. (2008). Perceptions of disorder: contributions of neighborhood characteristics to subjetive perceptions of disorder. Journal of Environmental Psychology, 28, 83-93. doi: 10.1016/j.jenvp.2007.08.003

García, C. (2012). La estructura de la percepción de la inseguridad pública. Liberabit. Revista de Psicología, 18(1), 37-44. http://www.redalyc. org/articulo.oa?id=68623931005

Huhn, S. (2008). La percepción social de la inseguridad en Costa Rica, El Salvador y Nicaragua. Revista de Ciencias Sociales (Cr), 4(122), 149-165. http://www.redalyc.org/ articulo.oa?id=15312992012

Kliksberg,B. (2008). ¿Cómo enfrentar la inseguridad en América Latina? Nueva sociedad, 15, 4-16. http://www.flacsoandes.edu.ec/agora/comoenfrentar-la-inseguridad-en-america-latinala-falacia-de-la-mano-dura

Medina, J. (2003). Inseguridad ciudadana, miedo al delito y policía en España. Revista Electrónica de Ciencia Penal y Criminología, 5 (3), 1-21. http://criminet.ugr.es/recpc/05/recpc05-03. pdf

Morquecho, A, C., y Vizcarra, L, R. (2008). Inseguridad pública y miedo al delito, un análisis de las principales perspectivas teóricas y metodológicas para su estudio. El estado de la cuestión sobre la inseguridad y el miedo al delito, 6, 1-20. http://dialnet.unirioja.es/ servlet/articulo codigo $=2888467$ 
Organismo de Investigación Judicial. (2015). Memoria Estadística 2015. Oficina de Planes y Operaciones, San José: Costa Rica. http:// pjenlinea.poderjudicial.go.cr/EstadisticasOIJ/ Documentos/Memoria\%20Estadistica\%20 2015.pdf

Pérez, F. (2012). Diferencias entre los usuarios de seis parques públicos en Barcelona según el nivel de seguridad percibida en el barrio. Athenea Digital, 12(1), 55-66. http://dialnet. unirioja.es/servlet/articulo?codigo $=4150770$

PNUD. (2009). Informe sobre Desarrollo Humano para América Central 2009-2010. Colombia: Programa de las Naciones Unidas para el Desarrollo. http://www.latinamerica.undp. org/content/rblac/es/home/library/human_ development/informe-sobre-desarrollohumano-para-america-central-2009-2010. html

Ralph,N.,Birks,M.,y Chapman, Y. (2015). The methodological dynamism of grounded theory. International Journal of Qualitative Methods, 14 (4), 1-6. doi:10.1177/1609406915611576

Salame, P. (2008). Informe sobre la Violencia en América Latina. Revista de Economía Institucional, 10 (18), 81-102. http:// www.scielo.org.co/scielo.php?pid=S012459962008000100004\&script=sci_abstract

Vilalta, C. (2010). El miedo al crimen en México. Estructura lógica, bases empíricas y recomendaciones iniciales de políticas públicas. Gestión y Política Pública, 19(1), 3-36. Recuperado de: http://www.redalyc. org/articulo.oa?id=13315701 
